\title{
Implementation of Design of Experiments in Development and Optimization of Transfersomal Carrier System of Tacrolimus for the Dermal Management of Psoriasis in Albino Wistar Rat
}

\author{
Ved Parkash ${ }^{*}$, Saurabh Maan¹, Vandana Chaudhary², Vikas Jogpal ${ }^{3}$, Girish Mittal ${ }^{3}$ and Vikas Jain ${ }^{4}$ \\ ${ }^{1}$ BS Anangpuria Institute of Pharmacy, Ballabgarh-Sohna Road, Faridabad, Haryana 121004 India. \\ ${ }^{2}$ School of Pharmacy, Pharmacy Australian Center of Excellence, University of Queensland, Queensland, Australia \\ ${ }^{3}$ School of Pharmaceutical Science, MVN University, $72 \mathrm{Km}$ mile stone, Delhi- Mathura Road, Aurangabad, Palwal 121105, India \\ ${ }^{4}$ Department of Pharmaceutics, JSS College of Pharmacy, JSS Academy of Higher Education and Research, SS Nagar, Mysore 570015, India
}

\begin{abstract}
The present study was aimed to deal with development and optimization of transfersomal system for enhancement of transdermal drug delivery of tacrolimus drug for the treatment of psoriasis. Transfersomes containing tacrolimus was prepared by rotary evaporation method using Box- Behnken design. The levels of the drug, phosphatidylcholine and sodium desoxycholate (independent variables) were varied to study the influence on particle size, \% entrapment efficiency and flux. The results of pharmacokinetic and pharmacodynamic studies proved that transfersomes were significantly superior in terms of drug permeation across the rat skin, with mean residence time of $52.58 \pm 3.62$ min. This was further confirmed by confocal laser scanning microscopic study. Transfersomes showed better antipsoriatic activities, compared to liposomes by virtue of better permeation through Wistar albino rat skin. Finally, it was concluded that the transfersomes accentuates the transdermal flux of tacrolimus and could be used for the management of psoriasis.
\end{abstract}

Keywords: Box-Behnken design; Psoriasis; Transfersomes; Tacrolimus; Design of experiments

\section{Introduction}

Psoriasis is a chronic inflammatory skin disorder typically characterized by erythematous plaques covered with silver or white scale on the surface and histologically characterized by proliferation of epidermis with parakeratosis, polymorphonuclear leukocyte infiltration in the stratum coreum, dilation of superficial blood vessels [1]. Whereas atopic dermatitis (AD) is a chronic, inflammatory skin disease characterized by an itchy, erythmatous and intensely pruritic rash with periods of exacerbation and remission [2]. For both the disease, exacerbation often requires treatment with topical corticosteroids, although effective, may be associated with several local and systemic adverse events, such as thinning of the skin and adrenal gland suppression. Patients' fears about the safety profile of topical corticosteroids also have important implications for adherence to treatment, and knowledge on differentiating weak preparations from strong preparations is poor [3]. Therefore, there is a need for an alternative treatment that is efficacious and free of the long-term side effects associated with corticosteroids.

The use of corticosteroids is regarded as a first line therapy for chronic patients, however the more prevalent and popular therapy is that of Tacrolimus and other drugs in this category which can be considered as second line therapy in this case.

Tacrolimus is a powerful macrolide immunosuppressant, isolated from the fermentation broth of Streptomyce tsukubaensis and has shown notable efficacy as a rescue or primary immunosuppressant therapy for immune-inflammatory conditions including atopic dermatitis. It inhibits early T-cell activation in gene transcription such as interleukin, which result from its inhibition of phosphatase (calcinuerin) in activated T-cells. It binds to cytoplasmic receptor FKBP-immunophilin and gain ability to associate with calcinuerin and inhibits its phosphatase activity resulting in inhibition of T-lymphocyte (i.e. immunosuppressant) and thus suppresses information [3]. However, Tacrolimus has proven to be effective for the treatment of skin diseases like atopic dermatitis or psoriasis. The main target for immunosuppressant drug Tacrolimus is the dermis with its lymphocytes. On the other hand, the formulation already available in the market are in ointment form which is again has disadvantage of greasy nature of dosage form and difficult to remove with low penetration across stratum corneum which gets extended in psoriatic skin. Therefore, it becomes a challenge to reach the dermis in sufficient active drug concentrations because of the high molecular size of Tacrolimus (822.05 $\left.\mathrm{gmol}^{-1}\right)$, structure and lipophilicity $(\log \mathrm{P} 3.96 \pm$ 0.83) [4] with an ointment base. Therefore, the objective become very clear to develop some alternative formulation which can carry the drug up to dermis layer of skin to improve the bioavailability and should be non-greasy with the advantage of ease of applicability.

Thus, it has been tried to formulate lipid vesicles because of their gaining interest over more than last two decades. Because of the low permeability capacity of conventional liposomes the researchers moved towards new facets in this science of drug delivery system and developed new classes of lipid vesicles. However innovative lipid vesicles have been introduced such as Transfersomes and ethosomes, which are presented superior skin penetration ability than conventional liposomes. The deformable liposomes were first introduced by Cevc and Blume in

'Corresponding author: Ved Parkash, Hyrdevangen 46 st tv 2400 Denmark København NV, Region Hovedstaden 2400 Denmark, Tel: + 4591665176; E-mail: vedps2011@gmail.com

Received November 19, 2018; Accepted December 12, 2018; Published December 19, 2018

Citation: Parkash V, Maan S, Chaudhary V, Jogpal V, Mittal G, et al. (2018) Implementation of Design of Experiments in Development and Optimization of Transfersomal Carrier System of Tacrolimus for the Dermal Management of Psoriasis in Albino Wistar Rat. J Bioequiv Availab 10: 98-106. 385. doi: 10.4172/09750851.1000385

Copyright: (C) 2018 Parkash V, et al. This is an open-access article distributed under the terms of the Creative Commons Attribution License, which permits unrestricted use, distribution, and reproduction in any medium, provided the original author and source are credited. 
Citation: Parkash V, Maan S, Chaudhary V, Jogpal V, Mittal G, et al. (2018) Implementation of Design of Experiments in Development and Optimization of Transfersomal Carrier System of Tacrolimus for the Dermal Management of Psoriasis in Albino Wistar Rat. J Bioequiv Availab 10 : 99-106. 385. doi: 10.4172/0975-0851.1000385

1992 as "Transfersomes" were the first generation vesicular system made up of phospholipids and edge activators and were reported to be able to penetrate skin carrying therapeutic concentrations of drugs $[5,6]$. They penetrates the skin membranes intact (5-10 times narrower than their own diameter), by squeezing themselves along with the intracellular sealing lipid of the stratum corneum under the influence of the trans-epidermal water-activity gradient and localize the drug at high concentrations without the significant loss in the deepest layers of the skin [7-9].

\section{Materials and Methods}

\section{Materials}

Tacrolimus (Tc) was received as a gift sample from Panacea Biotec Ltd., Lalru, Punjab, India. Phosphatidyl choline (Soya Lecichomax35) (PC) was purchased from Sonic Biochem Extractions Ltd., Indore, Madhya Pradesh, India. Sodium desoxycholate (SDC) was purchased from Otto Chemicals, Mumbai, India. Methanol (HPLC) was procured from Sisco Research Laboratories Pvt. Ltd., Mumbai, India. Rhodamine Red-X 1,2 dihexadecanoyl-sn-glycero-3-phosphoethanolamine trimethylammonium salt (RR) was purchased from Molecular Probes (Eugene, Oregon). Di-n-Propyl Disulfide was purchased from Chevron Phillips Chemical Company LLC, Texas, USA. Acetonitrile (HPLC), water (HPLC), Chloroform (AR) and Potassium Dihydrogen Orthophosphate was purchased from Rankem, New Delhi, India. Sodium hydroxide Pellets (AR) was purchased from Nice Chemicals Pvt. Ltd., Cochin. All the chemicals were of analytic grade reagents (AR).

Preparation of transfersomes using box- behnken design: Tacrolimus containing transfersomal formulations (TcTFs) were prepared by thin lipid film hydration method with some modifications [10], using Box- Behnken design. A mixture of PC and SDC containing tacrolimus was dissolved in a mixture of chloroform/ methanol (2: 1 $\mathrm{v} / \mathrm{v})$. The organic solution was added to a $50 \mathrm{ml}$ round bottom flask and the solvent was removed under reduced pressure temperature using rotary evaporator. The resulting film was then hydrated with $20 \mathrm{ml}$ phosphate buffer, pH 7.2 and the solution was allow to sonicate until ivory white color was observed. A three-factor three-level Box-Behnken design was employed to study the effect of independent variables on dependent variables as shown in Table 1.

Fifteen formulations were prepared according to the experimental design, the vesicle size, entrapment efficiency (EE \%), and transdermal flux obtained from the skin permeation study of tacrolimus transfersomes (TcTFs) are presented in Table 2.

Experimental design: A three -factor, three-level Box-Behnken design was used for exploring the quadratic response surfaces and construction of a second-order polynomial models using Quantum XL 2013 software (vesion 4.16) was used and a total of 15 experimental runs were constructed, for which the nonlinear computer-generated quadratic model is defined as:

$$
\begin{aligned}
& Y_{1}=126.38-1.8913 A+7.1463 B-16.908 C+2.235 A B+9.4975 A C \\
& -6.6225 B C+0.795 A^{2}-10.19 B^{2}-1.6825 C^{2}
\end{aligned}
$$

$$
\mathrm{Y}_{2}=76.283-5.3113 \mathrm{~A}+4.6413 \mathrm{~B}+14.768 \mathrm{C}+1.2 \mathrm{AB}-4.7925 \mathrm{AC}+
$$
$6.6875 \mathrm{BC}-7.4542 \mathrm{~A}^{2}-1.8442 \mathrm{~B}^{2}-3.0617 \mathrm{C}^{2}$

$$
\mathbf{Y}_{3}=568.6-15.298 \mathrm{~A}+11.17 \mathrm{~B}+67.89 \mathrm{C}+5.795 \mathrm{AB}+0.7 \mathrm{AC}+
$$

\begin{tabular}{|c|c|c|c|}
\hline \multirow{2}{*}{ Factor } & \multicolumn{3}{|c|}{ Level used, actual (coded) } \\
\hline & $\operatorname{Low}(-1)$ & Medium (0) & High (+1) \\
\hline \multicolumn{4}{|c|}{ Independent variables } \\
\hline $\mathrm{X} 1$ = Tacrolimus (mMol) & 4 & 7 & 10 \\
\hline X2 = Phosphatidyl choline (mMol) & 6 & 8 & 10 \\
\hline X3 = Sodium desoxycholate $(\mathrm{mMol})$ & 6 & 8 & 10 \\
\hline \multicolumn{4}{|c|}{ Dependent variables } \\
\hline \multicolumn{4}{|c|}{ Y1 = Vesicles size $(\mathrm{nm})$} \\
\hline \multicolumn{4}{|c|}{$\mathrm{Y} 2=\mathrm{EE} \%$} \\
\hline \multicolumn{4}{|c|}{$\mathrm{Y} 3=$ Flux $\left(\mu \mathrm{g} / \mathrm{cm}^{2} / \mathrm{hr}\right)$} \\
\hline
\end{tabular}
18.21BC $-43.4 \mathrm{~A}^{2}-3.57 \mathrm{~B}^{2}-11.165 \mathrm{C}^{2}$

Where $\mathrm{Y}_{1}=$ Vesicle size; $\mathrm{Y}_{2}=$ Entrapment efficiency; $\mathrm{Y}_{3}=$ Flux; $\mathrm{X}_{1}=$ Tacrolimus; $\mathrm{X}_{2}=$ Phosphatidylcholine; $\mathrm{X}_{3}=$ Sodium desoxycholate.

\begin{tabular}{|c|c|c|c|c|c|c|}
\hline \multirow{2}{*}{$\begin{array}{c}\text { Formulation } \\
\text { Code }\end{array}$} & \multicolumn{3}{|c|}{$\begin{array}{l}\text { Independent } \\
\text { variables }\end{array}$} & \multicolumn{3}{|c|}{ Dependent variables } \\
\hline & $\mathrm{X} 1$ & $\mathrm{X} 2$ & $\mathbf{X 3}$ & $Y 1(n=3 \pm S D)$ & $Y 2(n=3 \pm S D)$ & $Y 3(n=3 \pm S D)$ \\
\hline TcTFs 1 & 1 & 0 & 1 & $115.33 \pm 5.52$ & $70.82 \pm 4.31$ & $572.19 \pm 24.21$ \\
\hline TcTFs 2 & 0 & 1 & 1 & $99.23 \pm 4.14$ & $96.96 \pm 4.78$ & $647.28 \pm 29.34$ \\
\hline TcTFs 3 & 0 & 0 & 0 & $126.38 \pm 4.25$ & $74.31 \pm 5.20$ & $571.47 \pm 19.54$ \\
\hline TcTFs 4 & -1 & 0 & 1 & $103.32 \pm 4.87$ & $89.66 \pm 5.45$ & $598.45 \pm 30.44$ \\
\hline TcTFs 5 & -1 & 0 & -1 & $154.65 \pm 8.46$ & $51.13 \pm 4.23$ & $457.28 \pm 32.87$ \\
\hline TcTFs 6 & 0 & -1 & -1 & $116.54 \pm 6.83$ & $59.17 \pm 5.03$ & $496.87 \pm 25.73$ \\
\hline TcTFs 7 & 0 & 0 & 0 & $126.38 \pm 5.04$ & $78.06 \pm 3.65$ & $562.22 \pm 46.34$ \\
\hline TcTFs 8 & 0 & 0 & 0 & $126.38 \pm 3.40$ & $76.48 \pm 3.61$ & $572.11 \pm 23.25$ \\
\hline TcTFs 9 & -1 & 1 & 0 & $120.34 \pm 8.64$ & $77.23 \pm 6.34$ & $544.23 \pm 29.87$ \\
\hline TcTFs 10 & 0 & 1 & -1 & $147.77 \pm 5.48$ & $53.46 \pm 4.21$ & $481.87 \pm 15.41$ \\
\hline TcTFs 11 & 1 & 1 & 0 & $124.23 \pm 4.75$ & $67.64 \pm 6.52$ & $522.29 \pm 15.64$ \\
\hline TcTFs 12 & 1 & 0 & -1 & $128.67 \pm 4.65$ & $51.46 \pm 3.79$ & $428.22 \pm 24.70$ \\
\hline TcTFs 13 & 0 & -1 & 1 & $94.49 \pm 6.14$ & $75.92 \pm 5.04$ & $589.44 \pm 28.62$ \\
\hline TcTFs 14 & -1 & -1 & 0 & $114.21 \pm 4.33$ & $68.73 \pm 4.21$ & $532.56 \pm 32.28$ \\
\hline TcTFs 15 & 1 & -1 & 0 & $109.16 \pm 5.17$ & $54.34 \pm 6.36$ & $487.44 \pm 32.43$ \\
\hline
\end{tabular}

Table 1: Variables in Box-Behnken design for the preparation of Tacrolimus transfersomes.

Table 2: Observed response in Box-Behnken design for Tacrolimus transfersomal formulation (TcTFs).

Transfersomal size, shape and zeta potential: The vesicular diameter of the transfersomes was determined after storage at $4^{\circ} \mathrm{C}$ [11]. The vesicular size and size distribution of vesicles were determined by dynamic light scattering method, using a computerized inspection system Zetasizer 3000-HS (Malvern Instruments, Malvern, UK) [12]. For TEM imaging, the copper grids with a thin layer of carbon were loaded with TcTFs dispersion and allowed to dry under an IR-lamp. The sample was dried thoroughly and images were captured on a TEM Morgagni 268D (Fei Electon Optics, Eindhoven, the Netherlands).

Drug entrapment efficiency: The entrapment efficiency of transfersomal dispersion was determined by ultra-centrifuging the dispersion at $20,000 \mathrm{rpm}$ and $10^{\circ} \mathrm{C}$ for $30 \mathrm{~min}$ [13]. After centrifugation, $1 \mathrm{ml}$ of supernatant liquid was taken and diluted with $9 \mathrm{ml}$ phosphate saline buffer ( $\mathrm{pH}$ 7.4) and then the absorbance was measured using HPLC [14]. The analyses was carried out with a High Performance Liquid Chromatograph (Model-1260 infinity; Agilent Technologies, Santa Clara, California), equipped with an ultraviolet (UV) detector. Separation was achieved using zobax eclipse plus C-18 column (100 $\times 4.6 \mathrm{~mm}$, internal diameter $3.5 \mu \mathrm{m})$. The elution was carried out at a flow rate of $1 \mathrm{~mL} / \mathrm{min}$ using Methanol: water (80:20 v/v) as the mobile phase and maintaining the column temperature at $60^{\circ} \mathrm{C}$. Mobile phase was prepared and filtered daily by passing through a $0.45-\mu \mathrm{m}$ membrane filter, and then it was degassed at room temperature. The detection was carried out at $224 \mathrm{~nm}$ with a UV detector. The amount of entrapment drug expressed as a percentage was calculated from the following equation: 
Citation: Parkash V, Maan S, Chaudhary V, Jogpal V, Mittal G, et al. (2018) Implementation of Design of Experiments in Development and Optimization of Transfersomal Carrier System of Tacrolimus for the Dermal Management of Psoriasis in Albino Wistar Rat. J Bioequiv Availab 10: 99-106. 385. doi: 10.4172/0975-0851.1000385

\section{$\%$ Drug Entrapment Efficiency $=\left(\mathrm{W}_{\mathrm{T}}-\mathrm{W}_{\mathrm{F}}\right) / \mathrm{W}_{\mathrm{T}} \times 100 \%$}

Where, $\mathrm{W}_{\mathrm{T}}$ is the total amount of tacrolimus in transfersomal suspensions and WF is the free amount of Tacrolimus that was found in the supernatants.

Ex vivo skin permeation: The ex-vivo skin permeation of tacrolimus from transfersomes was studied using fabricated Franz diffusion cell with an effective permeation area and receptor cell volume of $1.0 \mathrm{~cm}^{2}$ and $15 \mathrm{~mL}$ respectively. After anesthetizing with chloroform adult Albino Wistar rat (200-250 gm) the animal was sacrificed and the hairs were trimmed carefully as short as possible using scissors without damaging or scratching the surface in order to ensure the integrity of the skin barrier and the prepared formulations of transfersomes each equivalent to $7 \mathrm{mg}$ of drug was applied onto the prepared skin facing the donor chamber. An aliquot of $500 \mu \mathrm{L}$ of samples was withdrawn at different time interval $(0,3,6,12,18$ and $24 \mathrm{~h})$ and replaced with same amount of fresh receiver vehicle to maintain the receptor phase volume as $15 \mathrm{ml}$ and analyzed for drug content by HPLC method and further extent of enhancement and enhancement ratio (ER) was calculated according to the following equation.

$$
\mathrm{ER}=\frac{\text { Steady state flux of formulation }}{\text { Steady state flux of control }}
$$

Equation 1: Determination of enhancement ratio.

Confocal laser scanning microscopy (CLSM): The depth of skin permeation was scanned by CLSM using the fluorescence signal of TcTFs at different skin depths. After completion of ex-vivo skin permeation study, the skin was removed and washed with distilled water and treated area was cut out and tested for probe penetration. The excised rat skin was positioned on the microscopic slide with the stratum corneum (SC) side facing the cover glass and observed under CLS microscope with fluorescence correlation spectroscope (Olympus Fluo View FV1000, Melville, New York) using an argon laser beam with excitation at $488 \mathrm{~nm}$ and emission at $590 \mathrm{~nm}$. Each skin sample was sliced in sections of $6-10 \mu \mathrm{m}$ thickness through the z-axis by CLSM. The TcTFs intensity and the permeated depth were detected by CLSM with Fluo View software.

In vivo pharmacokinetic and pharmacodynamics: The preclinical assessment of anti-psoriatic activity of the developed transdermal therapeutic system (TTS) was performed on experimentally psoriatic rats. Psoriasis was induced by painting a shaven animal area with din-propyl disulphide for 5 days [15]. The animals were divided into five groups (group A to E) of six animals each. Group A was taken as normal control. Psoriasis was induced in the remaining groups. Group B was served as psoriasis control and received no further treatment. Groups C, D, and E were subjected to placebo-TTS, tacrolimus-optimizedtransfersomes and tacrolimus-liposomes prepared by the same method which was followed by optimized transfersomal formulation, respectively. Each formulation was applied to the previously shaven abdominal area of rat skin. $500 \mu \mathrm{L}$ of blood samples were collected at $0,3,6,12,18$ and $24 \mathrm{~h}$ after the commencement of study. The sampling points 0 to $6 \mathrm{hr}$ were selected to determine the uptake of drug while 12, 18 and $24 \mathrm{hr}$ were used to assess the elimination of drug from the skin. At each sampling point the animal was anesthetized by chloroform and blood collected from tail vein for estimation of pharmacokinetic parameters. A pharmacodynamics study was also conducted in the same groups of animals. For this after the final dose administration, morphological changes of rat back skin, left and right ears were observed. The rat were then sacrificed and their ears of both sides (left and right) were cut off using a hole punching device $6 \mathrm{~mm}$ in diameter. The differences in mass and thickness between left and right ear were used to determine the induced ear swelling and skin tissues were excised and used to histological examination.

Assay of tacrolimus: The collected samples of blood from the rats were assayed with a modified HPLC method [16]. In brief, $150 \mu \mathrm{L}$ of blood samples were mixed with a $300 \mu \mathrm{L}$ of precipitation reagent, consisting of $50 \% \mathrm{v} / \mathrm{v}$ methanol, $30 \% \mathrm{v} / \mathrm{v}$ ethylene glycol, $20 \% \mathrm{v} / \mathrm{v}$ water and $100 \mathrm{mM}$ zinc sulphate. The samples were allowed to stand for 30 min at $4^{\circ} \mathrm{C}$, and then centrifuged at $14000 \mathrm{rpm}$ for 15 minutes. $180 \mu \mathrm{L}$ supernatant was fractionated by HPLC (Model-1260 infinity; Agilent Technologies, Santa Clara, California), equipped with an ultraviolet (UV) detector. Separation was achieved using zobax eclipse plus C-18 column $(100 \times 4.6 \mathrm{~mm}$, internal diameter $3.5 \mu \mathrm{m})$. The elution was carried out at a flow rate of $1 \mathrm{~mL} / \mathrm{min}$ using methanol: water (80: 20 $\mathrm{v} / \mathrm{v})$ as the mobile phase. After that treated blood sample of tacrolimus was injected to measure retention time of the drug that could be easily detected by using the UV spectrophotometric detector. $10 \mathrm{ngmL}^{-1}$ was the detection limit of this assay system with $10 \%$ coefficient of variance.

\section{Results}

\section{Effect of drug concentration}

From the results it was found that upon increasing tacrolimus concentration from $4 \mathrm{mg}$ to $7 \mathrm{mg}$ in the transfersomal system, the EE\% significantly increased from $51.13 \pm 4.23 \%$ (TcTFs5, with least concentration of sodium deoxycholate and phosphatidylcholine in optimum concentration) and nearly same when tacrolimus was used in optimum concentration $(7 \mathrm{mg}) 59.17 \pm 5.03 \%$ for TcTFs6 (Table 2). However, it has been observed that upon increasing the Tacrolimus concentration to $10 \mathrm{mg}$, the least entrapment efficiency of $54.34 \pm$ $6.36 \%$ was found (TcTFs 15) when the concentration of sodium desoxycholate was optimum and phosphatidylcholine was in least concentration.

\section{Vesicle size analysis}

The minimum vesicle size was observed as $94.49 \pm 6 \mathrm{~nm}$ for TcTFs 13 in which drug, was at optimum levels whereas phosphatidylcholine and desoxycholate at minimum and maximum levels respectively, whereas maximum vesicle size was observed as $154.65 \pm 8.46 \mathrm{~nm}$ for TcTFs 5 when sodium deoxycholate was minimum and phosphatidylcholine was used at optimum levels (Table 2). In the past, numerous researches have been reported that a decreased vesicle size is attributed to the presence of surfactant. The ultra-deformable liposomes (transfersomes) are also known as the first generation of elastic vesicles that contains an edge activator in their composition. The surfactants used in transfersome destabilize vesicular bilayer and increases bilayer elasticity by reducing the interfacial tension, hence leads to a decreased particle size. For the topical application a small particle size is of importance for the skin penetration and drug deposition [17]. The effect of independent variables on vesicle size has been represented in Figure 1.

\section{Effect of PC: EA ratio}

Entrapment efficiency (\%EE) is the fraction of percentage of the total drug that has incorporated into the transfersomes. The result shows maximum and minimum \%EE values obtained were $96.96 \pm$ $4.78 \%$ for TcTFs2 and $51.13 \pm 4.23 \%$ for TcTFs 5 , respectively (Table 2 ). Laisc and Fromherz gave a mechanistic picture of vesicle solubilisation/ formation and concluded that surfactant containing bilayers, at certain lipid/surfactant ratio, spontaneously un/curve and open or close 
Citation: Parkash V, Maan S, Chaudhary V, Jogpal V, Mittal G, et al. (2018) Implementation of Design of Experiments in Development and Optimization of Transfersomal Carrier System of Tacrolimus for the Dermal Management of Psoriasis in Albino Wistar Rat. J Bioequiv Availab 10: 99-106. 385. doi: 10.4172/0975-0851.1000385

to optimize edge tension [18]. When edge activators are used in the development of transfersomes in low concentration, growth in vesicle size occurred [19], whereas, further increase in their concentration may have led to pore formation in the bilayers and mixed micelles appeared to be coexisted with Transfersomes when edge activator concentration exceeded to $15 \%$. Consequently, lower drug entrapment due to the rigidity and smaller size of mixed micelles [20]. Effects of independent variables on \%EE are presented by three-dimensional graph in Figure 2.

The degree of transfersomal deformability is a crucial and unique characteristic parameter as it differentiates transfersomes from other lipid vesicles that are unable to cross intact skin by squeezing themselves up to 5-10 times of their size which is a unique advantage over any other lipid vesicles [20]. This membrane deformability is achieved by combining at least 2 lipophilic/ amphiphilic components $(\mathrm{PC}+\mathrm{EA})$, with sufficiently different packing characteristics, into a single bilayer. Transfersomes, consequently, undergo shape changes, whenever deformations are enforced by surrounding stress or space confinements, which minimizes the risk of vesicle rupture upon penetrating the skin pores.

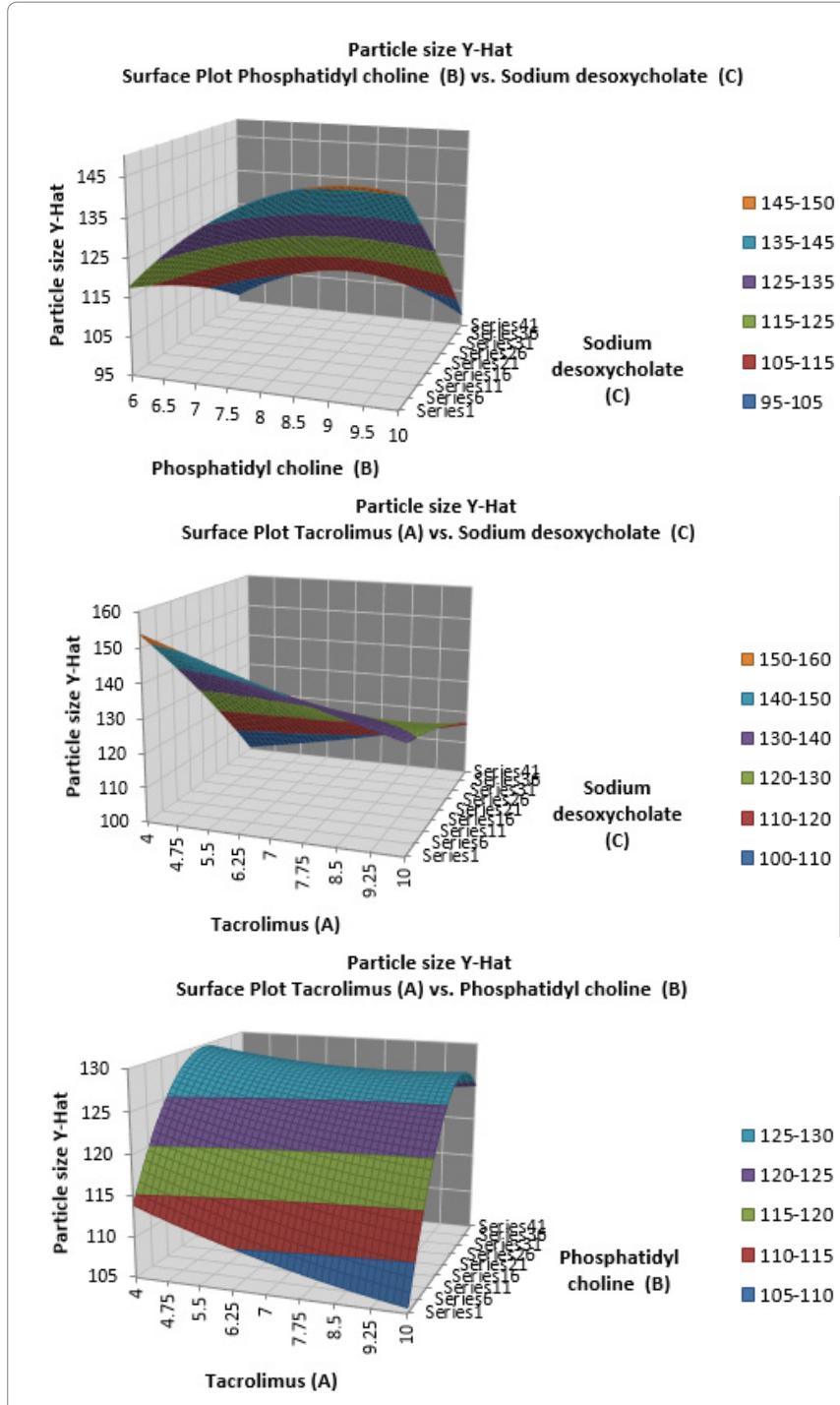

Figure 1: Effect of independent variables on vesicle size of transfersome containing Tacrolimus.

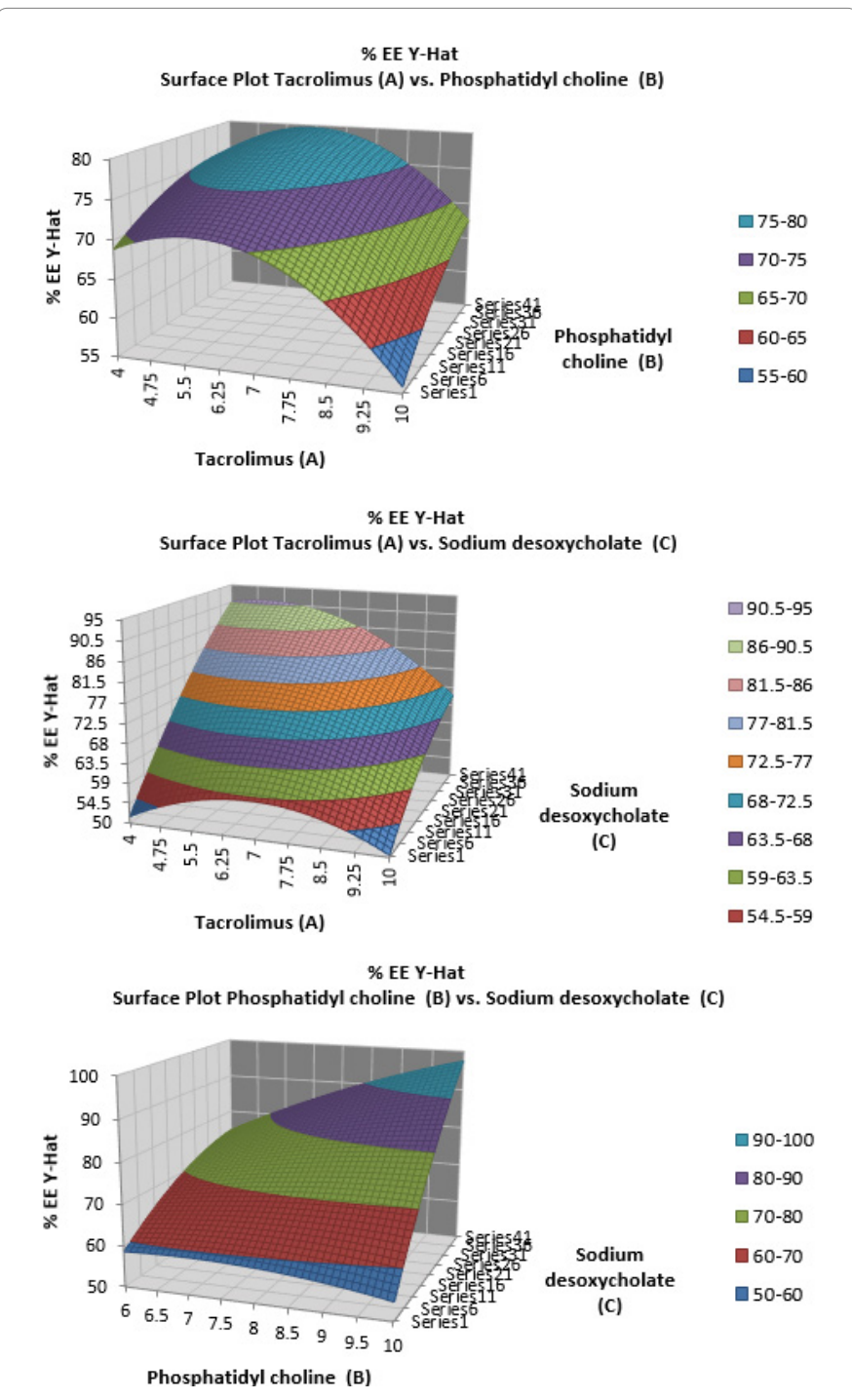

Figure 2: Effect of independent variables on Entrapment efficiency of Transfersome containing Tacrolimus.

\section{In vitro drug release/flux}

In vitro studies for the release of tacrolimus from transfersomes were performed as per the procedure detailed above. Fluxes from transfersomes at $24 \mathrm{~h}$ was first increased with increasing edge activator concentration (from 6 to $8 \mathrm{mMol}$ ) in the formulation and then decreased, a maximum flux $647.28 \pm 29.34 \mu \mathrm{g} / \mathrm{cm}^{2} / \mathrm{hr}$ was found for TcTFs 2 because in this formulation both lipid and edge activators were present in their maximum strength while a minimum flux 428.22 $\pm 24.70 \mu \mathrm{g} / \mathrm{cm}^{2} / \mathrm{hr}$ was observed for TcTFs12 that contain lipid at optimum level and edge activator at minimum. Effects of independent variables on flux are presented by three-dimensional graph in Figure 3.

\section{Confocal laser scanning microscopy}

By confocal laser scanning microscopy (CLSM), the extent of vesicle penetration into the stratum corneum was measured after topical application of two systems (i.e., optimized TcTFs and conventional liposomes each containing $0.03 \% \mathrm{RR}$ ). The results obtained have clearly defined the extent of transdermal penetration efficiency and potential of transfersomes as a drug carrier. The study shows a uniform distribution of drug throughout the stratum corneum, viable 
Citation: Parkash V, Maan S, Chaudhary V, Jogpal V, Mittal G, et al. (2018) Implementation of Design of Experiments in Development and Optimization of Transfersomal Carrier System of Tacrolimus for the Dermal Management of Psoriasis in Albino Wistar Rat. J Bioequiv Availab 10: 99-106. 385. doi: 10.4172/0975-0851.1000385

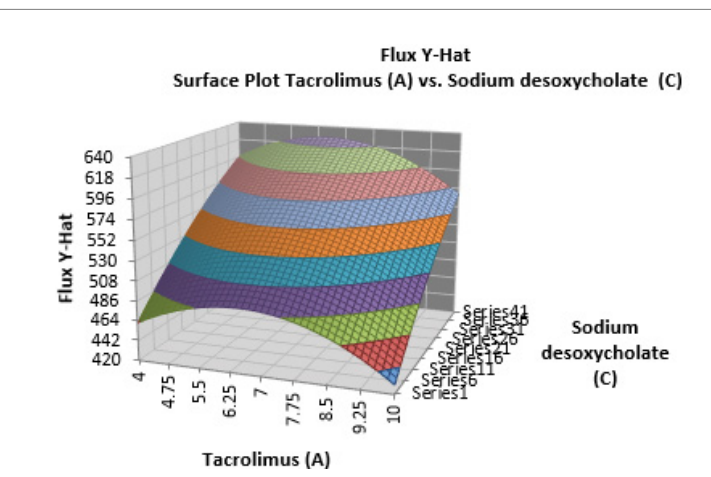

๑ 618-640

$\square 596-618$

$\square 574-596$

๑552-574

๑530-552

๑508-530

๑ 486-508

๑ 464-486

๑ 442-464

Flux Y-Hat

Surface Plot Phosphatidyl choline (B) vs. Sodium desoxycholate (C)

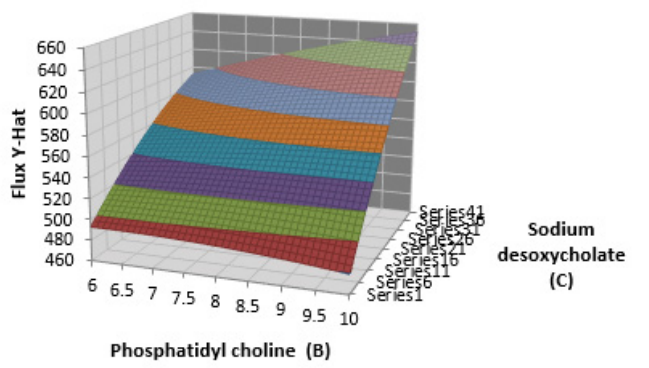

$\square 640-660$

$\square 620-640$

$\square 600-620$

$\square 580-600$

$\square 560-580$

घ40-560

๑520-540

ㅁ 500-520

- 480-500

Flux Y-Hat

Surface Plot Tacrolimus (A) vs. Phosphatidyl choline (B)

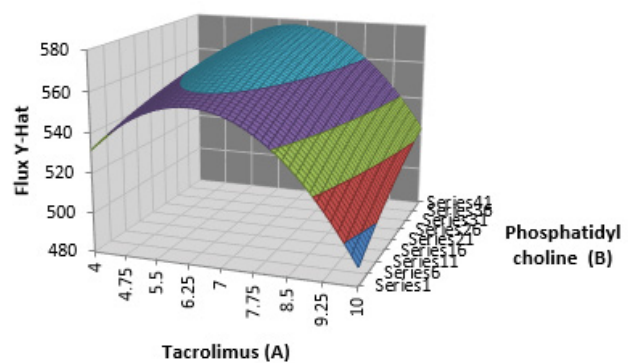

- $560-580$

- 540-560

- 520-540

- 500-520

-480-500

Tacrolimus (A)

Figure 3: Effect of independent variables on flux of transfersome containing Tacrolimus.

epidermis, and dermis with high fluorescence intensity Figure 4. In contrast, the rigid liposomes did not induce any change in the ultrastructure of cellular environment beneath the stratum corneum and being accumulated in the top most layer of the skin. Also, the fusion of conventional liposomes on upper layer of the stratum corneum might act as additional barrier for the diffusion of drugs across the stratum corneum and therefore inhibit skin permeation.

In support of this, histological studies of the skin were also performed in order to visualize the changes in the ultrastructure of stratum corneum and it was observed that no change in the ultrastructure of viable tissue (epidermis or dermis) could be observed after treatment with conventional liposomal formulation. The penetration from liposomal formulation was observed only to upper layer of skin (stratum corneum) and negligible in the deep Figure 4. In contrast, an enhanced delivery of probe (RR) in terms of depth (up to140 $\mu \mathrm{m}$ ) and quantity in dermis layer was observed using the transfersomal carrier. Thus, results showed the feasibility of using transfersomal formulation for delivering drugs into the deeper layers of skin or across the skin [21].

\section{In vivo pharmacokinetic and pharmacodynamics}

Psoriasis was induced in albino rats in order to examine the ability of the laboratory developed reservoir type dermal therapeutic system (DTS) of tacrolimus carrying tacrolimus- transfersomes- (TcTFs- 2) optimized and tacrolimus- liposomes as reservoir to provide a steadystate plasma concentration of the drug. The plasma drug concentration profiles of tacrolimus following the application of TcTFs- optimized and tacrolimus liposomes in rats are shown in Figure 5. A t-Test: Two-Sample Assuming Equal Variances was applied to compare the both formulations. The pharmacokinetic parameters evaluated were $\mathrm{C}_{\max }, \mathrm{T}_{\max }, \mathrm{t}_{1 / 2}, \mathrm{AUC}_{0-24}, \mathrm{AUC}_{0-\infty}, \mathrm{AUMC}_{0-24}, \mathrm{AUMC}_{0-\infty}$ and $\mathrm{MRT}$ is given in Table 3. The maximum drug concentration $\mathrm{C}_{\max }$ attained after optimized TcTFs formulation was observed as $1.91 \pm 1.1 \mathrm{ng} / \mathrm{mL}$ and
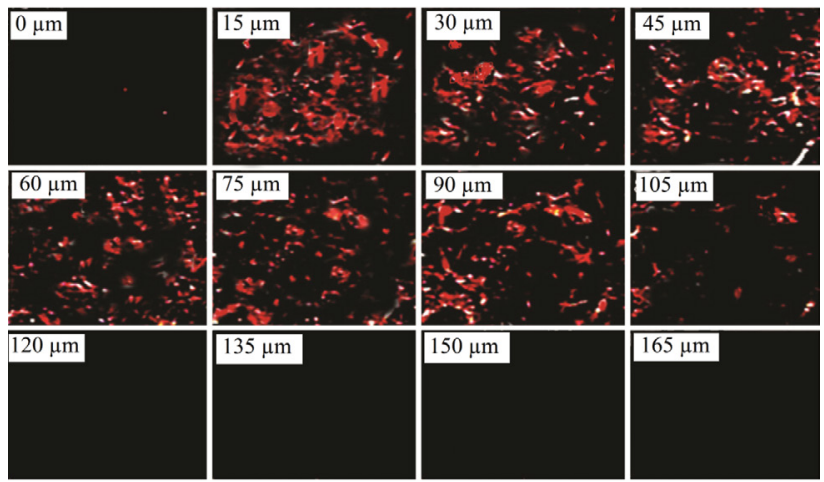

Figure 4: Confocal Laser Scanning Micrographs of TcTFs at various depth of skin.

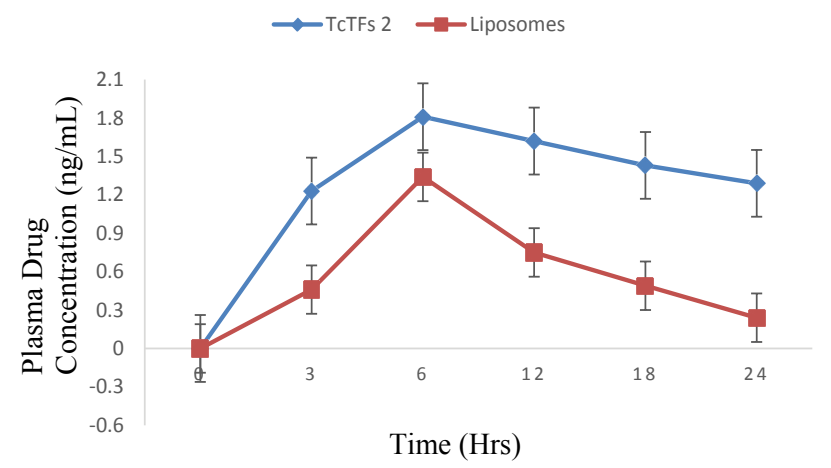

Figure 5: A plot between plasma drug concentration vs. time profile for transfersomes and liposomes.

\begin{tabular}{|c|c|c|}
\hline \multirow{2}{*}{ Pharmacokinetic parameters } & Transfersomes & Liposomes \\
\hline & TcTFs-2 $(n=6 \pm$ SD) & $(n=6 \pm S D)$ \\
\hline Cmax (ng/mL) & $1.91 \pm 1.1$ & $1.16 \pm 0.3$ \\
\hline $\operatorname{Tmax}(\mathrm{h})$ & $5.0 \pm 0.71$ & $6.0 \pm 1.84$ \\
\hline $\mathrm{t} 1 / 2(\mathrm{~h})$ & $34.65 \pm 4.36$ & $9.9 \pm 2.15$ \\
\hline AUC0-24 [(ng/mL)h] & $34.325 \pm 1.61$ & $15.66 \pm 2.34$ \\
\hline $\mathrm{AUCO}-\infty[(\mathrm{ng} / \mathrm{mL}) \mathrm{h}]$ & $98.825 \pm 9.07$ & $19.09 \pm 4.25$ \\
\hline AUMC0-24 [(ng/mL)h2] & $423.32 \pm 24.91$ & $163.48 \pm 11.63$ \\
\hline 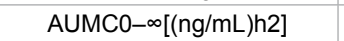 & $5196.32 \pm 131.54$ & $294.744 \pm 52.28$ \\
\hline MRT (h) & $52.58 \pm 3.62$ & $15.44 \pm 1.79$ \\
\hline
\end{tabular}

Table 3: Pharmacokinetic parameters of tacrolimus after single dermal application of transdermal therapeutic system to rats. 
Citation: Parkash V, Maan S, Chaudhary V, Jogpal V, Mittal G, et al. (2018) Implementation of Design of Experiments in Development and Optimization of Transfersomal Carrier System of Tacrolimus for the Dermal Management of Psoriasis in Albino Wistar Rat. J Bioequiv Availab 10: 99-106. 385. doi: 10.4172/0975-0851.1000385

$1.16 \pm 0.3 \mathrm{ng} / \mathrm{mL}$ for liposomal preparation which was significantly different compared to the liposomes $(\mathrm{p}<0.001)$. This difference in $\mathrm{C}_{\max }$ was due to the ultra-flexible nature of transfersomes as they squeeze themselves up to 5-10 times of their size while liposomes are failed to squeeze themselves. Concentration of tacrolimus decreased with increasing skin depth and it varied from 0.37 to 1.91 and 0.16 to 1.16 $\mathrm{ng} / \mathrm{mL}$, for transferosomes and liposomes respectively. The results comply with those observed by Krueger et al. and tacrolimus whole blood concentration was found to be less than $1 \mathrm{ng} / \mathrm{mL}$ [22].

The $\mathrm{T}_{\max }$ values in all the rats were higher for transfersomal application i.e. $5 \pm 0.7 \mathrm{hr}$ than the $5 \pm 1.8 \mathrm{hr}$ for liposomal formulations with a statistically significant difference $(\mathrm{p}<0.001)$ (Table 3$)$.

The value of elimination half-life $\left(\mathrm{T}_{1 / 2}\right) 34.65 \pm 4.3 \mathrm{hr}$ was found to be high for optimized TcTFs than $9.9 \pm 2.1 \mathrm{hr}$ for liposomes. The prolonged elimination half-life was contributed by the longer duration of absorption via the transdermal system [21]. The mean residence time was observed $52.58 \pm 3.62 \mathrm{~h}$ for transfersomes which is significantly high than the $15.44 \pm 1.79 \mathrm{~h}$ for liposomes (Figure 6).

\section{Drug quantification in three layers of skin}

In vivo skin retention studies were conducted in order to determine the distribution of tacrolimus in different layers of skin after the administration of TcTFs- 2 and liposomal formulations. It was observed that TcTFs facilitates a good penetration of tacrolimus then the liposomal formulation ( $\mathrm{p}<0.05)$. As it is already well known that $\mathrm{SC}$ is a rate limiting layer in percutaneous delivery system. Both the formulations showed almost same distribution of drug in SC. This shows that SC behaves like a saturated reservoir system. This occurs because of the lipid rich environment of SC then a slower permeation into deeper layers like epidermis and dermis would occurs. The revealed that the concentration of tacrolimus decreased gradually from SC to epidermis followed by dermis (SC $>$ epidermis $>$ dermis) and agree with the results obtained by Betz et al. [23]. The concentration of tacrolimus in epidermis and dermis was observed as $215.17 \pm 21.56 \mu \mathrm{g} /$ $\mathrm{cm}^{2}$ for TcTFs and $149.23 \pm 34.81 \mu \mathrm{g} / \mathrm{cm}^{2}$ for liposomal formulation. this was due to the rupturing of liposomes during squeezing through nano- pores that were much smaller than the liposomal vesicles because of their rigid structures hence they carry lesser amount of drug into the deeper layers of skin. The TcTFs overcome the nano- pores followed the natural water gradient across the deeper layers of skin because of their ultra flexibility and released a large amount of drug into epidermis and dermis as compared to liposomes [23].

\section{In vivo therapeutic efficacy}

The therapeutic efficacy of TcTFs was evaluated on psoriasis rat model followed by ear swelling, histopathological characterization and morphological appearance of treated rat skin with TcTFs- 2 and liposomal formulation Figure 7A-B. Showed change in ear swelling in terms of mass and thickness. cTFs showed a comparative depression $18.2 \mathrm{mg}$ to $10.1 \mathrm{mg}$ in ear swelling than $18.2 \mathrm{mg}$ to $15.2 \mathrm{mg}$ observed from liposoamal formulation. Similarly, TcTFsshown a remarkable decrease in ear thickness i.e. $8.5 \mathrm{~mm}$ to $6.6 \mathrm{~mm}$ compared to liposomal formulation i.e. $8.5 \mathrm{~mm}$ to $7.4 \mathrm{~mm}$.

The histopathological images of skin from this research comply with the available literature and it was shown that after a treatment of 7 days in injured skin started to be smother and better and also wounds started healing with ne hairs to grow on rough and dry skin with reduction in the skin thickness and inflammatory cell nucleus.

\section{Discussion}

Transfersomes were prepared by using Box- Behnken design by selecting three independent parameters at 3 levels to optimize the conditions of transfersomal preparation as given in Table 1 . They were extruded through a sandwich of polycarbonate membranes in order to reduce their size. As Transfersomes have an inherent tendency to be small and squeeze themselves [20]. In the vesicular formation the phosphatidylcholine concentration had little influence on drug loading ratio and encapsulation efficiency. However, it induced the rise of particle size and the appearance of flocculating constituent [24] depicted in Figure 6, so in TcTFs 10 phosphatidylcholine was at its lowest level, hence the size is too low. This indicates the fraction of phospholipid taking part in encapsulation was reduced upon increasing the total lipid concentration and similar observations were obtained by Mokhtar et al. who reported an optimum lipid concentration $[25,26]$. It has been conclude that small amounts of an EA in transfersomes increases the membranes flexibility, hence enabling them to pass more easily and readily through the pores of the polycarbonate filter during the vesicle preparation.

The vesicle size and entrapment efficiency has been given in Table

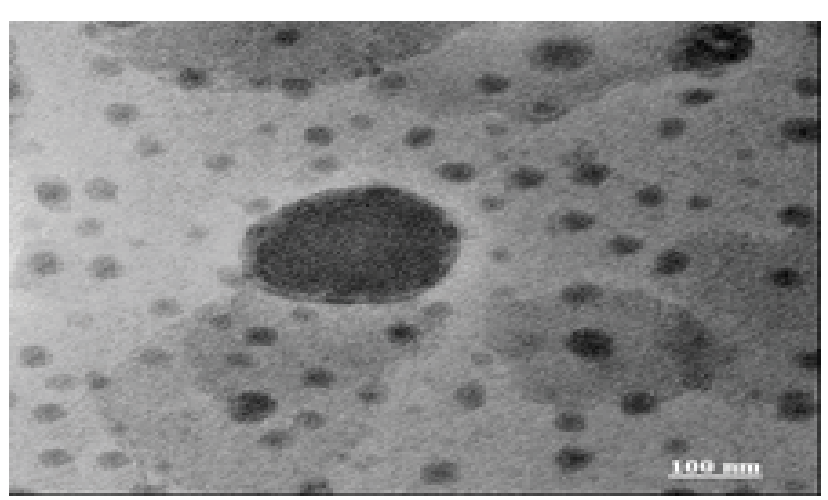

Figure 6: Transmission electron micrography following negative staining $(20,000 \times)$.

A

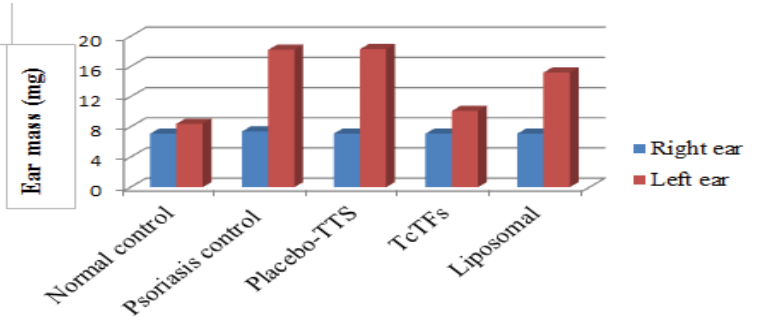

B

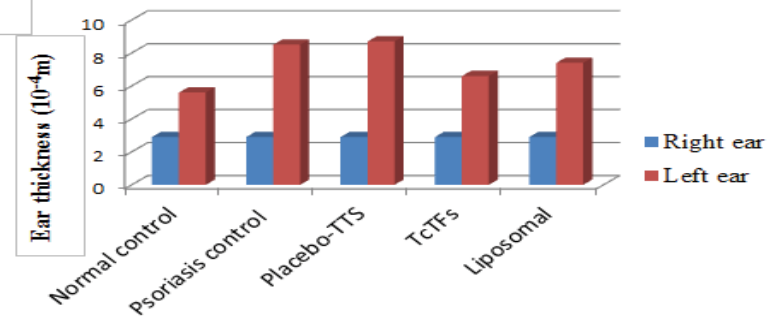

Figure 7: Ear mass $(A)$ and swelling $(B)$ of rat after last dose of different tacrolimus preparations $(n=6)$. 
Citation: Parkash V, Maan S, Chaudhary V, Jogpal V, Mittal G, et al. (2018) Implementation of Design of Experiments in Development and Optimization of Transfersomal Carrier System of Tacrolimus for the Dermal Management of Psoriasis in Albino Wistar Rat. J Bioequiv Availab 10: 99-106. 385. doi: 10.4172/0975-0851.1000385

2. The rotary evaporation-sonication method was optimized during preliminary studies and was found superior over vortexing as during vortexing it was visually observed that lipids were aggregate and adheres to the walls of vortexing vials and making hydration of vesicles difficult and was incapable of completely dispersing the lipids, hence, resulted in lumpy dispersions, difficultly homogenized and prone to rapid sedimentation and aggregation. On the other hand rotary evaporation- sonication method may influence the EE\% by formation of a thin film with a large surface area in the rotary evaporation method which enabled the complete hydration of vesicles. Thus, the rotary evaporation method was recommended despite the simplicity and less time consumption of the vortexing method.

The drug-loading ratio and entrapment efficiency have greatly influenced by the Phosphatidylcholine concentration. In this experiment, we found that the solvent mixture of ethanol and chloroform was selected to prepare TcTFs with phosphatidylcholine and other independent variables. It indicates that particle size increases with the rise of phosphatidylcholine concentration, while drug entrapment efficiency and potential raises first and then decreases afterwards. With the lower concentration of phosphatidylcholine the prepared sample was clear and transparent and diameter change was small. However, when higher Phosphatidylcholine concentration was used a turbid phenomenon was appeared and simultaneously drug entrapment efficiency and potential were reduced [24].

From Table 2, it has been observed that the entrapment efficiency is highly affected by the intrinsic properties of the surfactant, such as, hydrophilic lipophilic balance, chemical structure and phase transition temperature [27]. Means, the formulations containing surfactants of either low or high HLB value exhibits relative high entrapment efficiency, as the molecular geometry of hydrophobic non-ionic surfactants fulfills a proper critical packing parameter that incorporate a highly lipophilic drug into the vesicle bilayers $[28,29]$. However, the desoxycholate is a hydrophilic non-ionic surfactant with HLB value 16 able to form vesicles due to the presence of sufficient amount of cholesterol [30]. The entrapment efficiency is also governed by the length of the alkyl chain of surfactant as longer saturated alkyl chain showed higher entrapment efficiency [31]. Similar concept was discussed in various previous researches that the surfactants like span 85 , span 80 , tween 80 and sodium cholate and sodium deoxycholate with HLB values $1.8,4.3,15,16.7$ and 16.7 respectively and on the basis of these HLB values, the affinity for lipids was expected to be in the order of SP85> SP80> TW80> SC and SDC [25]. Transition temperature $(\mathrm{Tc})$ is another important factor that commonly helps to explain the vesicles entrapment patterns. Surfactants with high Tc are more likely in the ordered gel form forming less leaky bilayers, thus having higher entrapment efficiency, while surfactants with low Tc are more likely in the less ordered liquid form [30,32]. This may be due to the leakage of excess drug from the vesicular structure and other independent parameters used in transfersome preparations i.e. temperature conditions that directly affect the phopsho- linkage bonds of phosphatidylcholine that further inhibits the drug attachment. As upon rotary-evaporation sonication method, for generally the temperature is below the phase-transition temperature $(\mathrm{Tm})$, and it's difficult for the lipids to form a thin film above Tm. But the temperature at the time of film hydration must be more than Tm to guarantee the fluidity which is an important consideration for vesicular formation. This effect of independent variables on EE\% is presented by three-dimensional graph in Figure 3. According to Lopes et al. [33] the entrapment of drug occurs in both the bilayers and the aqueous compartment of the vesicles because with the increase of drug concentration saturation occurred in both lipid compartment and aqueous phase and the vesicles provides a limited entrapment capacity [34]. Only optimum ratio of lipid and edge activator could lead to flexibility of the bilayers membrane [35]. The above fact was also supported by Scognamiglio et al. [10] that encapsulation process of a lipophilic drug (tacrolimus) into lipid vesicles should be driven by drug solubility into the lipid phase and partition coefficient between lipid phase and aqueous solution. The high lipid solubility of tacrolimus together with the low water solubility should contribute to the high actual loading into the transfersomes. As the former researcher $[21,25]$ discussed we also observed that too low or too high concentration of edge activator (SDC) was not so beneficial in transfersomal delivery through skin. The observed results were also in agreement with published data $[6,20]$. The lipid membranes were more ordered and less leaky may be the possible cause for lower drug release when low edge activator concentrations were used, that impeded drug release. As the edge activators are only used to provide flexibility to the vesicles, but when they are used in higher concentrations the drug delivery decreases with decreased $\mathrm{EE} \%$ and also disrupt the lipid membrane that causes a leakage of entrapped drug from the vesicle [21]. Because of this behavior of edge activators the delivery of drug reduces, considering the possible carrier function of transfersomes and a mixed micelles could be formed that are less effective in transdermal drug delivery, because micelles are much less sensitive to a water activity gradient than transfersomes. In past this hypothesis was supported by Cevc et al. [17], who compared the penetration ability of different vesicular systems like transfersomes, liposomes, and mixed micelles by CLSM and concluded that mixed micelles were restricted to the top most part of the SC and that transfersomes penetrated to a deeper layers of skin $[36,37]$. From the pharmacokinetic studies it was concluded that the transfersomes represents a good utility as a drug carrier that can transport a lipophilic drug like tacrolimus with them. This happens because of the percutaneous absorption of target compounds from the transdermal formulation is affected by the manner of application. The percutaneous absorption of drugs can be classified into two pathways; i.e. the transepidermal route through the stratum corneum and second is the trans- appendageal route through the pilo-sebaceous unit or ecrine gland [38]. The ionized or hydrophilic compounds are greatly absorbed through the trans-appendageal route. On the other hand, the compounds with a partition coefficient of more than $1000 \mathrm{in} \mathrm{n-octanol/}$ water [39] system get absorbed through transepidermal route, and the partition coefficient of tacrolimus was more than 1000 [40]. From the results obtained it was suggested that tacrolimus would be absorbed through the transepidermal route. For the drugs absorbed through trans-epidermal pathway, the stratum corneum functions as a barrier for drug absorption and the absorption is enhanced with damaged skin [38].

\section{Conclusion}

In this study, tacrolimus containing transfersomes were compared with conventional liposomes prepared in the same way as transfersomes and were shown to enhance the permeation of Tacrolimus through the skin, suggesting that they are an excellent transdermal drug-delivery vehicle. The permeability of transfersomes was closely related to the composition of phosphatidylcholine and sodium deoxycholate while the other parameters like temperature and sonication time influences very little. The enhancement of permeation and release through the vesicle was attributed to the synergistic ability of transfersomes to act as a carriers, as well as, permeation enhancers. Hence, the transfersomes accentuates the transdermal flux of tacrolimus and create a window of opportunity in a well-controlled skin delivery of drugs that cause side effects upon administration by other routes and could be used for the management of psoriasis. 
Citation: Parkash V, Maan S, Chaudhary V, Jogpal V, Mittal G, et al. (2018) Implementation of Design of Experiments in Development and Optimization of Transfersomal Carrier System of Tacrolimus for the Dermal Management of Psoriasis in Albino Wistar Rat. J Bioequiv Availab 10: 99-106. 385. doi: 10.4172/0975-0851.1000385

\section{Acknowledgment}

Authors are highly thankful to the Director, B. S. Anangpuria Institute of Pharmacy, Faridabad, Haryana, India and Jamia Hamdard University, New Delhi, India for providing the laboratory facilities.

\section{References}

1. Yamamoto $T$ (2009) Topical Tacrolimus for Psoriasis. The Open Allergy Journal 2: 51-55

2. Pople PV, Singh KK (2010) Targeting tacrolimus to deeper layers of skin with improved safety for treatment of atopic dermatitis. Int J Pharm 398: 165-178.

3. Ashcroft DM, Dimmock P, Garside R, Stein K, Williams HC, et al. (2005) Efficacy and tolerability of topical pimecrolimus and tacrolimus in the treatment of atopic dermatitis: meta-analysis of randomized controlled trials. BMJ 330: 516

4. Goebel ASB, Neubert RHH, Wohlrab J (2011) Dermal targeting of tacrolimus using colloidal carrier systems. Int J Pharm 404: 159-168.

5. Cevc G, Blume G (1992) Lipid vesicles penetrate into intact skin owing to the transdermal osmotic gradients and hydration force Biochim Biophys Acta 1104: 226-32.

6. Jain S, Jain P, Umamaheshwari RB, Jain NK (2003) Transfersomes - a novel vesicular carrier for enhanced transdermal delivery: development, characterization, and performance evaluation. Drug Dev Ind Pharm 29: 10131026.

7. Cevc G, Blume G (2001) New, highly efficient formulation of diclofenac for the topical, transdermal administration in ultradeformable drug carriers, Transfersomes. Biochim Biophys Acta 1514: 191-205.

8. Maghraby GM, Barry BW, Williams AC (2008) Liposomes and skin: from drug delivery to model membranes. Eur J Pharm Sci 34: 203-222.

9. Maestrelli F, Gonzalez-Rodriguez ML, Rabasco AM, Ghelardini C, Mura P, et al. (2010) New "drug-in cyclodextrin-in deformable liposomes" formulations to improve the therapeutic efficacy of local anaesthetics. Int J Pharm 395: 222-231.

10. Scognamiglio I, Stefano DD, Campani V, Mayol L, Carnuccio R, et al. (2012) Nanocarriers for topical administration of resveratrol: A comparative study, Int J Pharm 440: 179-187.

11. Guo J, Ping Q, Sun G, Jiao C (2000) Lecithin vesicular carriers for transdermal delivery of cyclosporine. Int J Pharm 194: 201-207.

12. El Maghraby GM, Williams AC, Barry BW (2000) Skin delivery from ultradeformable liposomes: refinement of surfactant concentration. Int J Pharm 196: 63-74.

13. Malakar J, Sen SO, Nayak AK, Sen KK (2012) Formulation, optimization and evaluation of transfersomal gel for transdermal insulin delivery. Saudi Pharm J 20: 355-363.

14. Moffat AC, Osselton MD, Widdop B, Clarke EGC (2013) Tacrolimus: HPLC method development, Clarke's analysis of drugs and poisons: in pharmaceuticals, body fluids and postmortem material, Pharmaceutical Press, $4^{\text {th }}$ edition, p: 2278.

15. Skuri J, Oroli N, Kolari D, Diki D, Benkovi V, et al. (2011) Effectivity of flavonoids on animal model psoriasis - thermographic evaluation, Periodicum Biologorum 113: $457-463$

16. Okabe H, Hashimoto $\mathrm{Y}$, Inui $\mathrm{K}$ (2000) Pharmacokinetics and bioavailability of tacrolimus in rats with experimental renal dysfunction. J Pharm Pharmacol 52 : 1467-1472.

17. Chen G, D Li, Y Jin, Zhang W, Teng L, et al. (2013) Deformable liposomes by reverse-phase evaporation method for an enhanced skin delivery of (b)catechin. Drug Dev Ind Pharm 40: 260-265.

18. Simões SI, Marques CM, Cruz ME, Cevc G, Martins MB, et al. (2004) The effect of cholate on solubilisation and permeability of simple and protein-loaded phosphatidylcholine/sodium cholate mixed aggregates designed to mediate transdermal delivery of macromolecules. Eur J Pharm Biopharm 58: 509-519.

19. Van den Bergh BA, Wertz PW, Junginger HE, Bouwstra JA (2001) Elasticity of vesicles assessed by electron spin resonance, electron microscopy and extrusion measurements. Int J Pharm 217: 13-24.
20. Kumar A, Pathak K, Bali V (2012) Ultra-adaptable nanovesicular systems: a carrier for systemic delivery of therapeutic agents. Drug Discov Today 17: 1233-1241.

21. Ahad A, Aqil M, Kohli K, Sultana Y, Mujeeb M, et al. (2012) Formulation and optimization of nanotransferosomes using experimental design techniques for accentuated transdermal delivery of valsartan. Nanomedicine: Nanotechnology Biology, and Medicine 8: 237-249.

22. Krueger GG (2007) Pharmacokinetics of tacrolimus following topical application of tacrolimus ointment in adult and pediatric patients with moderate to severe atopic dermatitis. J Drugs Dermatol 6: 185-193.

23. Betz G, Imboden R, Manidis G (2001) Interaction of liposome formulations with human skin in vitro. Int J Pharm 229: 117-129.

24. Zheng WS, Fang XQ, Wang LL, Zhang YJ (2012) Preparation and quality assessment of itraconazole transfersomes. Int J Pharm 436: 291- 298.

25. El Zaafarany GM, Awad GA, Holayel SM, Mortada ND (2010) Role of edge activators and surface charge in developing ultradeformable vesicles with enhanced skin delivery. Int J Pharm 397: 164-172.

26. Mokhtar M, Sammour OA, Hammad MA, Megrab NA (2008) Effect of some formulation parameters on flurbiprofen encapsulation and release rates of niosomes prepared from proniosomes. Int J Pharm 361: 104-111.

27. El Laithy HM, Shoukry O, Mahran LG (2011) Novel sugar esters proniosomes for transdermal delivery of vinpocetine: Preclinical and clinical studies. Eur $\mathrm{J}$ Pharm Biopharm 77: 43-55

28. Fang JY, Yu SY, Wu PC, Huang YB, Tsai YH, et al. (2001) In vitro skin permeation of estradiol from various proniosome formulations. Int $\mathrm{J}$ Pharm 215: 91-99.

29. Alsarra IA, Bosela AA, Ahmed SM, Mahrous GM, (2005) Proniosomes as a drug carrier for transdermal delivery of ketorolac. Eur J Pharm Biopharm 59: 485-490.

30. Uchegbu IF, Vyas SP (1998) Non-ionic surfactant based vesicles (niosomes) in drug delivery. Int J Pharm 172: 33-70.

31. Guinedi AS, Mortada ND, Mansour S, Hathout RM (2005) Preparation and evaluation of reverse-phase evaporation and multilamellarniosomes as ophthalmic carriers of acetazolamide. Int J Pharm 306: 71-82.

32. Mohammed AR, Weston N, Coombes AG, Fitzgerald M, Perrie Y, et al. (2004) Liposome formulation of poorly water soluble drugs: optimisation of drug loading and ESEM analysis of stability. Int J Pharm 285: 23-34.

33. Lopes LB, Scarpa MV, Silva GVJ, Rodrigues DC, Santilli CV, et al. (2004) Studies on the encapsulation of Diclofenac in small unilamellar liposomes of soya phosphatidylcholine. Colloids Surf B Biointerfaces 39: 151-158.

34. Ning MY, Guo YZ, Pan HZ, Yu HM, Gu ZW, et al. (2005) Preparation and evaluation of proliposomes containing clotrimazole. Chem Pharm Bull 53: 620-624.

35. Gupta PN, Mishra V, Rawat A, Dubey P, Mahor S, et al. (2005) Non-invasive vaccine delivery in transfersomes, niosomes and liposomes: a comparative study. Int J Pharm 293: 73-82.

36. EI Maghraby GM, Williams AC, Barry BW (2000) Oestradiol skin delivery from ultradeformable liposomes: refinement of surfactant concentration. Int J Pharm 196: 63-74.

37. Hiruta Y, Hattori Y, Kawano K, Obata Y, Maitani Y, et al. (2006) Novel ultradeformable vesicles entrapped with bleomycin and enhanced to penetrate rat skin. J Control Release 113: 146-154.

38. Barry BW (1983) Skin transport, in Dermatological Formulations. Percutaneous Absorption. Marcel Dekker Inc, New York. 95-126.

39. Raykar PV, Fung MC, Anderson BD (1988) The role of protein and lipid domains in the uptake of solutes by human stratum corneum. Pharm Res 5: 140-150.

40. Hane K, Fujioka M, Namiki Y, Kitagawa T, Kihara N (1992) Physico-chemical properties of (-)-1R,9S,12S,13R,14S,17R,18E,21S,23S,24R,25S,27R)-17allyl-1,14-dihydroxy-12-[(E)-2-[(1R,3R,4R)-4-hydroxy-3-methoxycyclohexyl]1-methylvinyl]-23,25-dimethoxy-13,19,21, 27-tetrametyl-11,28-dioxa-4azatricyclo[22.3.1.04,9]octacos- 18-ene-2,3,10,16-tetrone hydrate (FK-506) lyakuhinKenkyu 23: 33-43. 